\title{
Nature, Strength, and Consequences of Indirect Adsorbate Interactions on Metals
}

\author{
A. Bogicevic, ${ }^{1, *}$ S. Ovesson, ${ }^{2}$ P. Hyldgaard, ${ }^{2}$ B. I. Lundqvist, ${ }^{2}$ H. Brune, ${ }^{3}$ and D. R. Jennison ${ }^{4}$ \\ ${ }^{1}$ Chemistry Department, Ford Research Laboratory, MD 3083 SRL, Dearborn, Michigan 48121-2053 \\ ${ }^{2}$ Department of Applied Physics, Chalmers University of Technology and Göteborg University, S-412 96 Göteborg, Sweden \\ ${ }^{3}$ Institut de Physique Expérimentale, Ecole Polytechnique Fédérale de Lausanne, CH-1015 Lausanne, Switzerland \\ ${ }^{4}$ Surface and Interface Sciences Department, Sandia National Laboratories, Albuquerque, New Mexico 87185-1421
}

(Received 31 January 2000)

\begin{abstract}
Atoms and molecules adsorbed on metals affect each other indirectly even over considerable distances. Via systematic density-functional calculations, we establish the nature and strength of such interactions, and explain for what adsorbate systems they critically affect important materials properties. This is verified in kinetic Monte Carlo simulations of epitaxial growth, which help rationalize a number of recent experimental reports on anomalously low diffusion prefactors.
\end{abstract}

PACS numbers: 68.35.Fx, 68.35.Bs, 68.35.Ja

Besides forming direct chemical bonds at short separations, atoms and molecules interact indirectly over large distances via relaxations in the lattice of substrate atoms on which they are adsorbed. In metals, polarization of the electron gas gives rise to a supplementary form of indirect adsorbate interactions [1-5]. Because almost nothing is known about the relative and absolute strengths of these long-ranged elastic and electronic interactions for real metals, it is unclear in general how they modify adsorbate behavior and affect materials properties.

Microscopic studies that have addressed this problem are scarce [6-9]. Recent experimental measurements have suggested that long-range interactions strongly influence adsorbate ordering on metals, and have shown that traditional analyses fail $[6,10,11]$. While such accounts are invaluable for quantifying adsorbate interactions, direct studies are needed to pin down the relative roles of elastic versus electronic effects and, most importantly, address a fundamental question: when must we worry about indirect interactions?

Many materials properties are modeled with implicit assumptions about these interactions. For instance, a common way of experimentally determining adsorbate mobilities is to measure the island density and then via mean-field nucleation theory or kinetic simulations deduce the corresponding activation energy and diffusion prefactor [12]. This standardized way of measuring diffusion rates has pointed out systems with anomalously low (by many orders of magnitude) prefactors [12].

In this Letter, we address these issues from first principles. For two electronically quite different metals, calculations within density-functional theory directly reveal the magnitude, oscillations, and falloff of the electronic part with the distance between adatoms at binding sites and in transition states. These numbers cannot be predicted from simple models. Then relaxation is allowed so that the elastic component is isolated and its effects examined. By comparing the induced perturbations in the potentialenergy landscape with surface corrugation and adsorbate binding, we predict for what materials systems indirect interactions are maximally important. These perceptions are corroborated by kinetic Monte Carlo simulations of epitaxial growth which show a large increase in island density when indirect interactions are taken into account. An immediate consequence of this particular result is that it offers an explanation to a series of recent reports on anomalously low diffusion prefactors.

For reasons outlined below, we focus our study on two metal systems: $\mathrm{Al} / \mathrm{Al}(111)$ and $\mathrm{Cu} / \mathrm{Cu}(111)$. The calculations are based on density-functional theory (DFT) $[13,14]$, using a pseudopotential method, as implemented in the VASP code [15]. For the exchange-correlation functional, the local-density approximation (LDA) [16] is used for $\mathrm{Al}$, and the generalized gradient approximation (GGA) [17] for $\mathrm{Cu}$. The one-electron wave functions are expanded in a plane-wave basis with an energy cutoff of 9.0 (17.2) Ry for $\mathrm{Al}(\mathrm{Cu})$, using ultrasoft Vanderbilt pseudopotentials [18]. The Brillouin zone is sampled according to the MethfesselPaxton scheme [15]. The Kohn-Sham equations are solved self-consistently, and the atomic structure optimized until forces on all unconstrained atoms are less than $0.03 \mathrm{eV} / \AA$, which yields convergence down to the $1 \mathrm{meV}$ level.

To reduce image interactions, the periodic supercells used in the calculations span at least twice the maximum adsorbate-adsorbate distance examined in a specific direction. For Al, our (111) supercell consists of $14 \times 4 \times 6$ (336) atoms; for $\mathrm{Cu}$ we employ a $12 \times 4 \times 4$ cell containing 192 atoms (computer memory constraints prohibit bigger cells for $\mathrm{Cu}$ ). Above an additional adsorbate layer [19], there is $\simeq 9$ (13) $\AA$ of vacuum for $\mathrm{Al}(\mathrm{Cu})$. The Brillouin zone is sampled using a $6 \times 2(3 \times 1) \mathbf{k}$-point mesh for $\mathrm{Al}(\mathrm{Cu})$. The dense sampling and large cells yield excellent energy convergence; adatom diffusion barriers and dimer binding energies for $\mathrm{Al}$ on both unrelaxed and relaxed surfaces are within $2 \mathrm{meV}$ of previous calculations using a $6 \times 5 \times 6$ atom supercell and $6 \times 6 \mathrm{k}$-point mesh [20].

To separate electronic and elastic adsorbate interactions, all calculations are performed at two levels of relaxation. In one case, the slab (no adsorbates) is first fully relaxed, 
and the atomic coordinates then kept frozen in subsequent calculations. In the other case, all atoms are allowed to relax, save for the bottom two layers which in both cases are fixed at bulk coordinates. In both instances, two atoms are adsorbed on top of the slab, and (always) allowed to fully relax. For $\mathrm{Al} / \mathrm{Al}(111)$, one atom is placed in an hcp site (the preferred binding site at low coverage), and the other atom is placed at consecutive hcp, bridge, and fcc sites along the $\langle 110\rangle$ direction. In the $\mathrm{Cu} / \mathrm{Cu}(111)$ calculations, one atom is placed in an fcc site, and the other in alternating bridge and fcc sites along the $\langle 110\rangle$ direction. The maximum adatom-adatom separation is 17 (13) $\AA$ for $\mathrm{Al}(\mathrm{Cu})$, slightly less than half the length of the 41 (31) $\AA$ supercell so that image interactions should never exceed half the interaction energy at maximum separation. Saddle points for atomic diffusion are located by mapping out the total energy on a dense grid near bridge sites; in this case one or two of the lateral adsorbate coordinates are locked at each point on the mesh. Part of the supercell is illustrated in Fig. 1.

To set the stage and establish part of the unperturbed potential-energy surface, we first consider self-diffusion of isolated adatoms. The computed activation energies
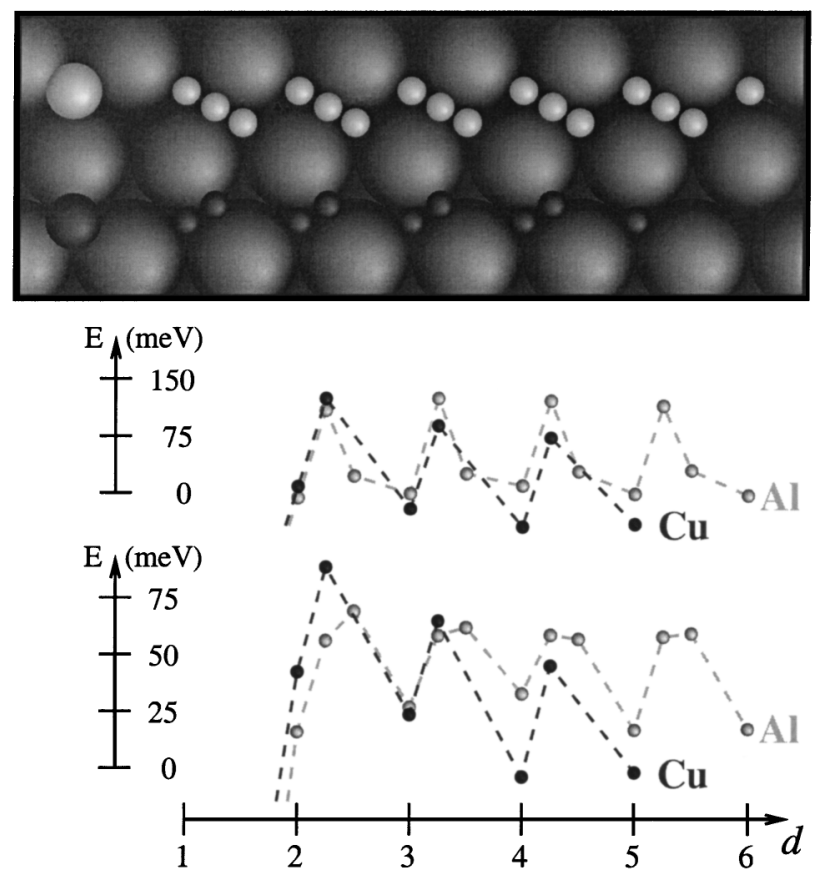

FIG. 1. Top view of adsorption geometries in the DFT calculations. One adatom is placed at its preferred binding site (hcp for $\mathrm{Al}$ and $\mathrm{fcc}$ for $\mathrm{Cu}$; midsize circles), and another atom is then placed at successive binding sites and saddle points (tiny circles) along the $\langle 110\rangle$ direction (half the length of the Al supercell is shown). The binding energy is defined as $E=$ $E_{1}^{1}+E_{1}^{2}-E_{0}-E_{2}$, where the subscript denotes the number of adatoms in the cell and the superscript identifies the individual atomic positions, and is shown as a function of adsorbate separation $d$ in terms of lattice sites. Both frozen (middle graph) and relaxed (bottom graph) cases are truncated at short separations to enhance resolution.
$E_{d}$ are given in Table I. Note that the elastic substrate response considerably smooths out the surface corrugation, which greatly facilitates the diffusion process in lowering the activation energy by a factor of 3 for both $\mathrm{Al} / \mathrm{Al}(111)$ and $\mathrm{Cu} / \mathrm{Cu}(111)$ [21].

We start out by identifying three distinct interaction regimes according to adsorbate separation: (i) At minimal interatomic separations, direct electronic interactions prevail, and localized chemical dimer bonds are formed $[20,23]$. This interaction falls off exponentially, and is therefore very short ranged [1]. At its extreme, it can effectively extend up to a couple of lattice sites, and form a "denuded" zone on weakly corrugated surfaces, where adatom pairs are unstable with respect to dimerization (cf. the issue of capture radii in Refs. $[8,23,24]$ ). (ii) At larger separations, adsorbate interactions are predominantly indirect, and mediated in three ways: via electrostatic (dipoledipole) and elastic fields, which both decay monotonically with separation $d$ as $1 / d^{3}$, and via oscillatory Friedeltype quantum corrections [1,2]. (iii) The most long-ranged form of indirect interaction is found for metals with a partially filled surface band (assuming a near-spherical Fermi surface), where the Friedel-type interaction energy decays asymptotically as $-\sin \left(2 q_{\mathrm{F}} d\right) / d^{2}[1,2,5], q_{\mathrm{F}}$ being the in-plane Fermi wave vector. In the absence of a surface band, the same interaction falls off much faster, as $\cos \left(2 q_{\mathrm{F}} d\right) / d^{5}[1,2]$.

To contrast indirect adsorbate interactions in the presence or absence of a surface band, $\mathrm{Cu} / \mathrm{Cu}(111)$ and $\mathrm{Al} / \mathrm{Al}(111)$ are studied. The $\mathrm{Cu}(111)$ surface band should produce asymptotic oscillations with a period $15 \AA$ - the shortest among the noble metal surfaces [5], and thus potentially best to investigate in a supercell geometry. The $\mathrm{Al}(111)$ surface has no occupied surface-band states and should provide a reference.

The DFT computed adatom-adatom interaction energetics for $\mathrm{Al} / \mathrm{Al}(111)$ and $\mathrm{Cu} / \mathrm{Cu}(111)$ are illustrated in Fig. 1 and summarized in Table I. Since short-range interactions are well understood [20,23], we focus on regimes (ii) and (iii). With adatom separation $d$, we find variations in the total energy at both binding sites and saddle points. The fact that the range of variations in the interatomic potential due to indirect interactions are still there in their full extent even when elastic response is frozen (Fig. 1, Table I) indicates that indirect interactions are mainly electronic in this regime. It is interesting to note though that elastic substrate

TABLE I. DFT values for the atomic diffusion barrier in the case of an isolated adatom $\left(E_{d}\right)$ and the range of variation in barriers produced by the presence of another adatom $\left(E_{d}^{*}\right)$ on the frozen and relaxed substrates. All values are in meV.

\begin{tabular}{ccccc}
\hline \hline & \multicolumn{2}{c}{ Frozen } & \multicolumn{2}{c}{ Relaxed } \\
System & $E_{d}$ & $E_{d}^{*}$ & $E_{d}$ & $E_{d}^{*}$ \\
\hline $\mathrm{Al} / \mathrm{Al}(111)$ & 115 & $110-126$ & 42 & $24-53$ \\
$\mathrm{Cu} / \mathrm{Cu}(111)$ & 134 & $110-146$ & 50 & $41-69$ \\
\hline \hline
\end{tabular}


response is still very important, in that it lowers the atomic diffusion barriers by smoothening out the surface corrugation. This effect triples the $\left(E_{d}^{*}-E_{d}\right) / E_{d}$ ratios $-E_{d}^{*}$ $\left(E_{d}\right)$ being the atomic diffusion barriers with (without) adsorbate interactions - and thereby increases the relative magnitude of indirect electronic interactions.

Although these supercells are extraordinarily large for a systematic first-principles study, they still capture only part of the indirect interaction curve. We certainly note a long-ranged variation for $\mathrm{Cu}$, but it is not possible to discern how well its periodicity agrees with half the Fermi wavelength $\pi / q_{\mathrm{F}}=15 \AA$ measured by Crommie et al. [25]. A density-of-states examination does show a surface band, although it is clear that its position and characteristics are affected by slab-thickness convergence problems as well as intrinsic shortcomings of LDA/GGA. A graphic indication of the importance of indirect interactions on $\mathrm{Cu}(111)$ is given by recent scanning-tunneling microscopy (STM) observations of long-range ordering of sulfur atoms with a periodicity of $15 \AA$ [26].

We now turn to discuss the strength of indirect interactions, and their impact on surface morphology. At elevated temperatures, relative adsorbate binding energies dictate the surface structure $[8,24,27]$. We find a binding energy variation of about $17 \mathrm{meV}$ for $\mathrm{Al}$, and about $46 \mathrm{meV}$ for $\mathrm{Cu}$ adatoms due to indirect interactions. At low temperatures, kinetic limitations control the surface morphology $[8,24,27]$. The computed total-energy variations at binding sites and saddle points translate into separation and direction dependent atomic diffusion barriers (Fig. 1, Table I). For Al, the activation energy assumes values of $24-53 \mathrm{meV}$, to be contrasted against the nominal $42 \mathrm{meV}$ of isolated adatoms. For $\mathrm{Cu}$, the barrier is $41-69 \mathrm{meV}$, compared with $50 \mathrm{meV}$ for isolated adatoms. For both systems, the "perturbations" are therefore almost as large as the atomic diffusion barrier itself.

With these results in mind, one might wonder how it is possible in general to understand and model many materials systems while completely neglecting long-ranged interactions. The key to understanding this question is to compare the perturbations stemming from indirect interactions with other characteristic energies. For many systems, mRy variations in the potential-energy landscape are small compared with adsorbate bond energies and surface diffusion barriers, and we therefore expect them to only weakly perturb adsorbate interactions. Conversely, it is also clear that indirect interactions should be important for adsorbatemetal systems with weak ad-ad bonds and/or a weakly corrugated surface. Examples here include, but are not limited to, homogeneous and heterogeneous $M / M(111)$ and $R / M(111)$ systems, where $M$ can be $\mathrm{Al}, \mathrm{Cu}, \mathrm{Ag}, \mathrm{Au}$, and $R$ any of the rare gases, and judging from recent reports $[10,26]$, possibly chalcogens and halogens as well. In these cases, the perturbation of the energy landscape that results from indirect adsorbate interactions is comparable to the corrugation of the landscape itself, and (except for pure metal systems) of the same magnitude as chemical adsorbate bonds.

Indirect interactions are consequently expected to have a strong effect on the diffusion kinetics and aggregation for this type of adsorbate-metal systems. To test these ideas on laboratory time and length scales, we perform $a b$ initio kinetic Monte Carlo (KMC) simulations [28] of the low-temperature growth of $\mathrm{Al}(111)$ and $\mathrm{Cu}(111)$. The only input to these calculations is a set of previously DFT-computed activation energies [24] and the current long-range perturbations to the energy landscape. Possible angular dependencies of interaction energies are unknown, and thus neglected, and the interaction between more than two adatoms is described by pairwise summation. Theoretical studies by Einstein [1] suggest that the latter is a good approximation beyond the shortest adatom separations, as corroborated in a recent STM study [11]. A detailed account of these calculations will be presented elsewhere; a summary follows below.

A representative indicator of surface morphology is the island density. In the case of $\mathrm{Al}$, with a deposition flux $F=0.01$ monolayer/s (ML/s), coverage $\Theta=5 \%$, and substrate temperature of $25 \mathrm{~K}$, the computed indirect interactions roughly triple the island density (compared with the absence of long-range interactions). Efforts to better account for the asymptotic decay in the KMC simulations further augment this effect, but an accurate quantitative estimate thereof cannot be made at present because of high sensitivity of nucleation dynamics to activation energy fluctuations at separations beyond our DFT data. For $\mathrm{Cu} / \mathrm{Cu}(111)(25 \mathrm{~K}, F=0.01 \mathrm{ML} / \mathrm{s}, \Theta=10 \%)$, indirect interactions lead to a fivefold increase of the island density, see Fig. 2. The enhanced nucleation results from the fact that the diffusion barriers are notably larger for associating adatoms than dissociating them. Assuming pairwise additive interactions, such a repulsion inhibits attachment to existing islands and leads to an increased island density compared with the same system devoid of long-range interactions.

The fact that indirect long-ranged adsorbate interactions can drastically increase the island density is very interesting for experimentally determined adsorbate diffusivities.

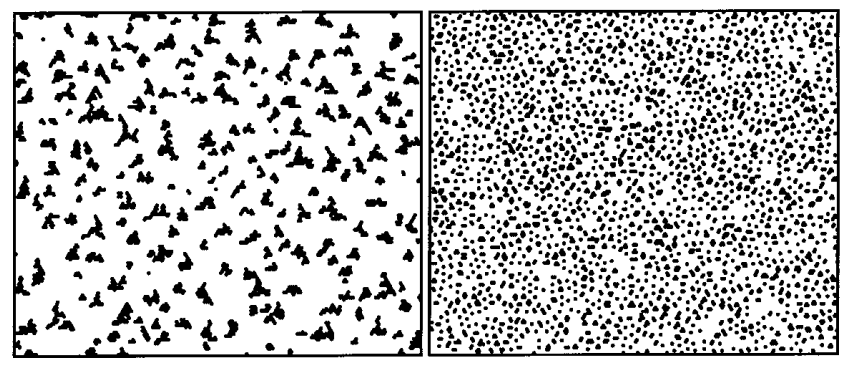

FIG. 2. KMC-computed surface morphology $\left(500 \times 425 \AA^{2}\right)$ of $\mathrm{Cu} / \mathrm{Cu}(111)$ at $T=25 \mathrm{~K}, F=0.01 \mathrm{ML} / \mathrm{s}, \Theta=10 \%$ without (left) and with (right) taking into account the DFT-calculated long-range interactions [29]. 
If long-range interactions are unaccounted for, the island density will be too low, and artifically low diffusion prefactors will be deduced from mean-field nucleation theory or kinetic simulations. For example, if the island density decreases by a factor of 10 because long-range interactions are neglected, the deduced prefactor will typically appear 3 orders of magnitude smaller than it really is. Over the last few years, a series of anomalously low (by up to 10 orders of magnitude) diffusion prefactors have been reported for weakly corrugated systems [12]. According to our analysis above, this particular class of systems is exactly where indirect interactions should be important. It is thus quite possible, even likely, that such anomalies are unphysical [30], and a mere consequence of neglecting to account for indirect interactions in the analysis of island density data. While we demonstrate the significance of such interactions for island nucleation, even more long-ranged interaction energies should be considered in order to determine whether adsorbate interactions alone can account for the anomalies in STM island densities, something which at present is unattainable with the DFT-KMC method.

In summary, we have determined the nature and strength of indirect adsorbate interactions in two different systems from first principles, and shown these to significantly alter the binding and motion of otherwise isolated adsorbates. We explain where it is important to take such long-ranged interactions into account, and demonstrate via kinetic simulations the strong effects they can have on surface morphology. These results suggest that reports of anomalously low diffusion prefactors may be an artifact of neglecting indirect interactions in analyses of experimental island density data.

We respectfully acknowledge critical comments by Ted Einstein. This study was partly inspired by accounts of ongoing research by Kristen Fichthorn and Matthias Scheffler, and a preprint was provided to us after the submission of our manuscript. One of us (D. R. J.) acknowledges the encouragement of Ernst Bauer. All DFT calculations were performed at Sandia, a multiprogram laboratory operated by Sandia Corporation, a Lockheed Martin Company, for the United States Department of Energy under Contract No. DE-AC04-94AL85000. This work was partially supported by a Laboratory Directed Research and Development project and by the Swedish Research Council for Engineering Sciences (TFR). Allocation of computer time at the UNICC facilities at Chalmers University of Technology is gratefully acknowledged.

\footnotetext{
*Corresponding author.

Email address: abogicev@ford.com
}

[1] T. L. Einstein and J. R. Schrieffer, Phys. Rev. B 7, 3629 (1973); T. L. Einstein, Surf. Sci. Lett. 75, 161 (1978); Handbook of Surface Science, edited by W. N. Unertl (Elsevier, Amsterdam, 1996), Vol. 1, p. 577.

[2] K. H. Lau and W. Kohn, Surf. Sci. 75, 69 (1978).

[3] K. H. Lau and W. Kohn, Surf. Sci. 65, 607 (1977).

[4] T. B. Grimley, Proc. Phys. Soc. London 90, 751 (1967); 92, 776 (1967).

[5] P. Hyldgaard and M. Persson, J. Phys. Condens. Matter 12, L13 (2000).

[6] T. T. Tsong, Phys. Rev. B 6, 417 (1972); Atom-Probe Field Ion Microscopy (Cambridge University Press, Cambridge, 1990).

[7] G. L. Kellogg, Surf. Sci. Rep. 21, 1 (1994).

[8] H. Brune, Surf. Sci. Rep. 31, 121 (1998).

[9] S. C. Wang and G. Ehrlich, Phys. Rev. Lett. 70, 41 (1993); F. Watanabe and G. Ehrlich, J. Phys. Chem. 95, 6075 (1991); 96, 3191 (1992).

[10] S. Renisch et al., Phys. Rev. Lett. 82, 3839 (1999).

[11] L. Österlund et al., Phys. Rev. Lett. 83, 4812 (1999).

[12] H. Brune et al., Phys. Rev. B 52, 14380 (1995); B. G. Briner et al., Science 278, 257 (1997); B. Fischer et al., Phys. Rev. Lett. 82, 1732 (1999); J. V. Barth et al., Phys. Rev. Lett. 84, 1732 (2000).

[13] P. Hohenberg and W. Kohn, Phys. Rev. 136, B864 (1964).

[14] W. Kohn and L. J. Sham, Phys. Rev. 140, A1133 (1965).

[15] G. Kresse and J. Hafner, Phys. Rev. B 47, 558 (1993); 49, 14251 (1994); 54, 11169 (1996).

[16] J. P. Perdew and A. Zunger, Phys. Rev. B 23, 5048 (1981).

[17] J. P. Perdew et al., Phys. Rev. B 46, 6671 (1992).

[18] D. Vanderbilt, Phys. Rev. B 32, 8412 (1985).

[19] The artificial electric field induced by one-sided adsorption is very weak; when compensated by a dipole layer in the vacuum, energy differences change by $\lesssim 10 \mathrm{meV}$ and (more importantly) by the same energy amount for different adsorption sites.

[20] A. Bogicevic, Phys. Rev. Lett. 82, 5301 (1999).

[21] The previously reported bridge-fcc degeneracy for $\mathrm{Al} / \mathrm{Al}(111)$ [22,23] turns out to be a purely elastic effect; with a frozen substrate the degeneracy is lifted, and the fcc site is markedly favored over the near-bridge site.

[22] R. Stumpf and M. Scheffler, Phys. Rev. Lett. 72, 254 (1994).

[23] A. Bogicevic, P. Hyldgaard, G. Wahnström, and B.I. Lundqvist, Phys. Rev. Lett. 81, 172 (1998).

[24] A. Bogicevic, J. Strömquist, and B. I. Lundqvist, Phys. Rev. Lett. 81, 637 (1998).

[25] M.F. Crommie, C.P. Lutz, and D. M. Eigler, Nature (London) 363, 524 (1993).

[26] E. Wahlström et al., Appl. Phys. A 66, S1107 (1998).

[27] S. Ovesson, A. Bogicevic, and B. I. Lundqvist, Phys. Rev. Lett. 83, 2608 (1999).

[28] A. F. Voter, Phys. Rev. B 34, 6819 (1986).

[29] For diffusion processes taking place at the island perimeters, we use the DFT-computed barriers for $\mathrm{Al} / \mathrm{Al}(111)$ from Ref. [24], although at this temperature such processes only marginally affect the island density.

[30] C. Ratsch and M. Scheffler, Phys. Rev. B 58, 13163 (1998). 